\title{
Isolated Septic Arthritis of the Hip Due to Fusobacterium Nucleatum in An Immunocompetent Adult: A Case Report and Review of the Literature
}

\author{
Masanori Nishi ${ }^{1}$, Yasushi Yoshikawa ${ }^{1}$, Yasutaka Kaji ${ }^{1}$, Satoshi Okamoto ${ }^{1}$, Katsunori Inagaki ${ }^{1}$
}

\section{Learning Point of the Article:}

Fusobacterium nucleatum can be the cause of septic arthritis, even in an immunocompetent patient, requiring surgical debridement and appropriate antibiotic therapy.

\section{Abstract}

Introduction: Hip septic arthritis is more common in children than in adults. Staphylococcus aureus and Streptococcus spp. are commonly found in association with septic joints. In contrast, Fusobacterium nucleatum septic arthritis in adults is extremely rare. To the best of our knowledge, only five cases have been reported in the literature in English, and three of them were cases of periprosthetic joint infection. We report a rare case of hip septic arthritis due to $F$. nucleatum in an immunocompetent adult.

Case Presentation: A 56-year-old Asian man with a history of bilateral Perthes' disease and mild alcoholic liver disease presented to our hospital complaining of worsening right hip pain and difficulty in walking for the previous 3 weeks. On presentation, his temperature was $38.7^{\circ} \mathrm{C}$, and laboratory results showed a white blood cell count of 19200 cells $/ \mu \mathrm{L}$ and a C-reactive protein level of $43.56 \mathrm{mg} / \mathrm{dL}$. Hip movements were limited due to pain. Contrast-enhanced computed tomography and magnetic resonance imaging showed fluid retention, suggesting infection. F. nucleatum was detected in the culture test from joint aspirate. Surgical drainage was performed 3 times in combination with antibiotherapy. Finally, we performed two-stage total hip arthroplasty, and the post-operative course was uneventful without implant loosening or infection relapse.

Conclusion: The patient had a history of Perthes' disease and had hip osteoarthritis, which may have contributed to the development of hip septic arthritis. We treated this rare case of hip septic arthritis due to F. nucleatum with two-stage revision surgery and antibiotherapy. Clinicians should be aware that F. nucleatum could be the etiologic agent of hip septic arthritis in an immunocompetent patient.

Keywords: Septic Arthritis, Hip joint, Fusobacterium nucleatum, Adult

\section{Introduction}

Fusobacterium species are Gram-negative anaerobic bacillus. They have been isolated from mainly oral and dental infectious diseases, brain abscesses, bacteremia, and soft-tissue infections $[1,2]$. To date, a few authors have reported the isolation of Fusobacterium species from bone and joint infections [3]. Septic arthritis is an infection of a synovial joint due to the pathogenic inoculation of the joint (which may occur either directly or through the hematogenous route) and involves a potential risk of high morbidity and mortality [4]. Hip septic arthritis is more common in children than in adults, and

\begin{tabular}{|c|}
\hline Access this article online \\
\hline Website: \\
www.jocr.co.in \\
\hline DoI: \\
10.13107/jocr.2021.v11.04.2142 \\
\hline
\end{tabular}

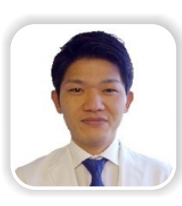

Dr. Masanori Nishi

Staphylococcus aureus and Streptococcus spp. are often found in association with septic joints [5]. There have been very few reports on septic arthritis caused by Fusobacterium nucleatum in adults $[2,6,7,8,9]$. Here, we report a very rare case of hip septic arthritis due to F. nucleatum in a male adult.

\section{Case Presentation}

A 56-year-old man with a history of bilateral hip osteoarthritis due to Perthes' disease and mild alcoholic liver disease presented to our hospital complaining of worsening right hip

\section{Author's Photo Gallery}

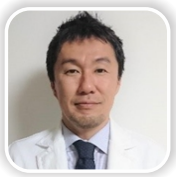

Dr. Yasushi Yoshikawa

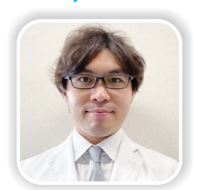

Dr. Satoshi Okamoto

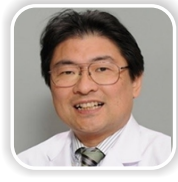

Dr. Katsunori Inagaki

'Department of Orthopaedic Surgery, Showa University School of Medicine, Hatanodai, Shinagawa-ku, Tokyo, Japan.

Department of Orthopaedic Surgery, Showa University School of Medicine, Hatanodai, Shinagawa-ku, Tokyo, Japan.

E-mail: m.nishi83@gmail.com

Journal of Orthopaedic Case Reports | pISSN 2250-0685 | eISSN 2321-3817 | Available on www.jocr.co.in | doi:10.13107/jocr.2021.v11.i04.2142 This is an Open Access article distributed under the terms of the Creative Commons Attribution Non-Commercial License (http://creativecommons.org/licenses/by-nc/3.0) which permits unrestricted non-commercial use, distribution, and reproduction in any medium, provided the original work is properly cited. 


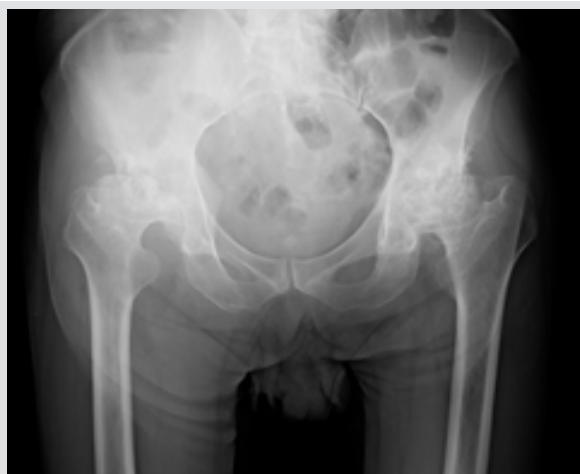

Figure 1: Radiograph of the anteroposterior pelvis showing a narrowjoint space at the bilateral hip joint.

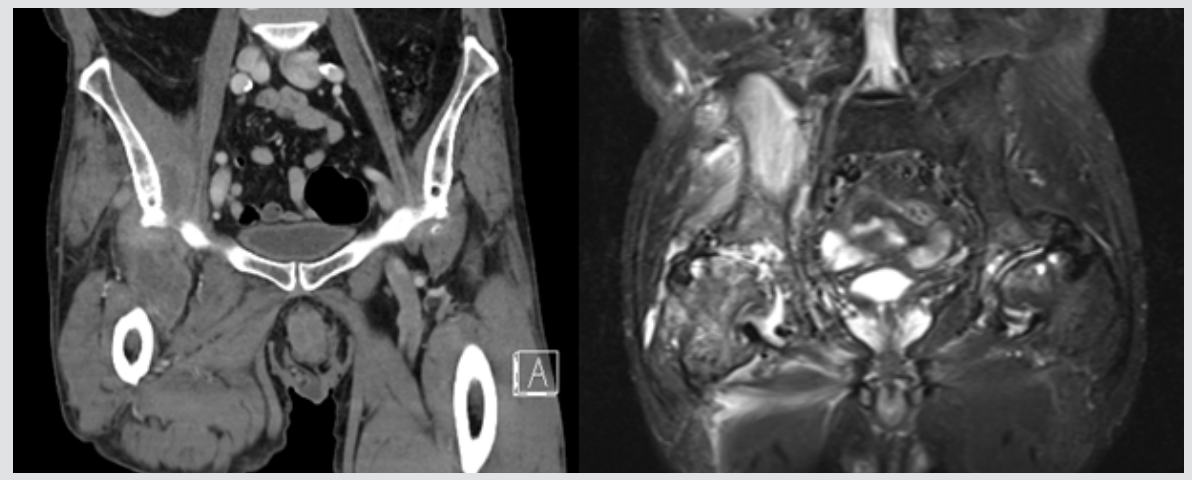

Figure 2: Contrast-enhanced coronal computed tomography showing fluid retention from the iliac muscle to the region around the right hip. Magnetic resonance imaging showing A T2 high-intensity lesion around the right hip and iliac muscle. pain and difficulty in walking for the 3 previous weeks. On presentation, his temperature was $38.7^{\circ} \mathrm{C}$, and laboratory results revealed a white blood cell count of $19.2 \times 103$ cells/L and C-reactive protein level of $43.56 \mathrm{mg} / \mathrm{dL}$. The right hip experienced extreme pain on mobilization. Radiographs showed bilateral hip osteoarthritis due to Perthes' disease (Fig. 1). Contrast-enhanced computed tomography showed fluid retention from the iliac muscle to around the right hip, and magnetic resonance imaging of the right hip was suggestive of septic arthritis (Fig. 2). Three-phase bone scintigraphy showed increased activity in all phases (Fig. 3).

The right hip was aspirated and specimens were sent for pathological and microbiological examination. We performed surgical drainage (on day 1). During the first phase of the procedure, we took three samples for analysis and all intraoperative biopsy samples were positive for F. nucleatum. One week after the first surgery (on day 8), we performed femoral head resection and added antibiotics to the cement spacer. In total, arthrotomy with debridement was performed 3 times (on days 1, 8, and 35). On admission, parenteral antibiotic treatment was started with meropenem. After confirming that the culture was positive for F. nucleatum, we started treatment with intravenous cefmetazole for 21 days. After the third procedure, we changed antibiotics to intravenous cefazolin and clindamycin due to a poor inflammatory response. Finally, we successfully performed two stage total hip arthroplasty (THA) on day 75 (Fig. 4). The post-operative course was uneventful with the oral administration of minocycline. The patient showed no evidence of implant loosening or infection relapse during the 3 years of follow-up.

\section{Discussion}

F. nucleatum is a normal inhabitant of the oral cavity, female genitals, and gastrointestinal tract. It is often identified as the cause of oral infections, skin and soft tissue infections, and intraabdominal abscesses [6]. However, F. nucleatum septic arthritis in adults is extremely rare. To the best of our knowledge, only five cases of F. nucleatum septic arthritis in adults have been reported in the literature in English, and three of them were cases of periprosthetic joint infection (Table 1) [2, $6,7,8,9]$. While there are two reports of knee septic arthritis, this is the first case report of hip septic arthritis caused by F. nucleatum in adults.

Lemierre disease is an oropharyngeal infection caused by F. necrophorum, which is complicated by sepsis, thrombosis of the internal jugular vein, and multiple metastatic infections, including septic arthritis [10]. Williams et al. [11] reported a case of Lemierre disease caused by F. nucleatum. In our case, no history of recent oropharyngeal infection or dental portal of entry was noted on clinical examination. There is some evidence supporting the idea that bacteria from a distant site such as the oral cavity could spread to the joints in cases of rheumatoid arthritis or osteoarthritis [6]. This patient had hip

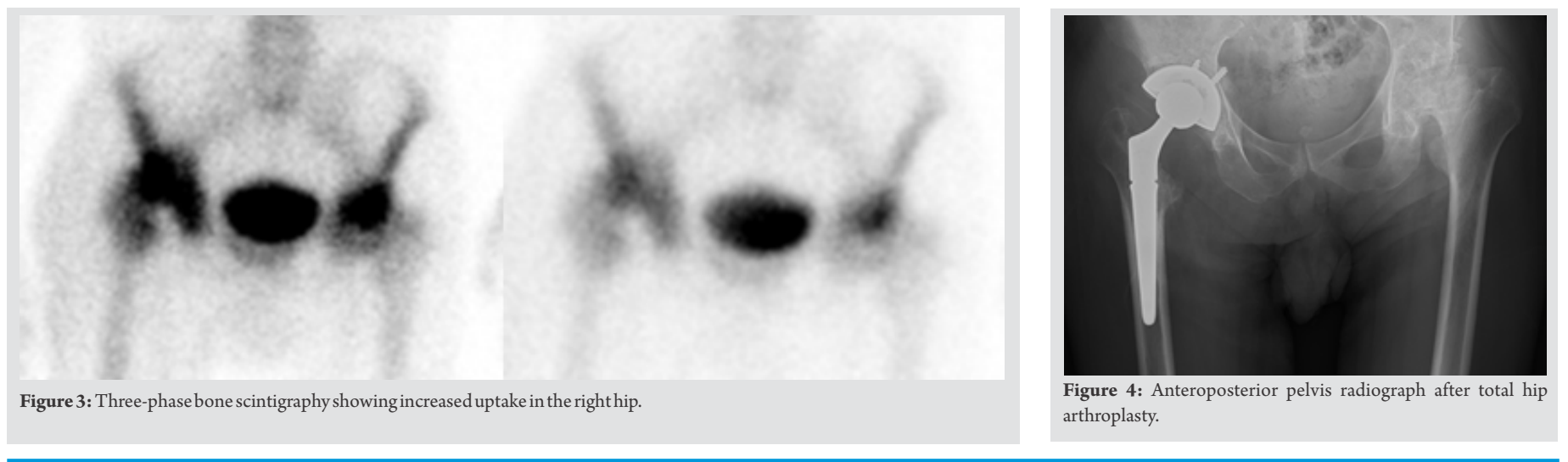




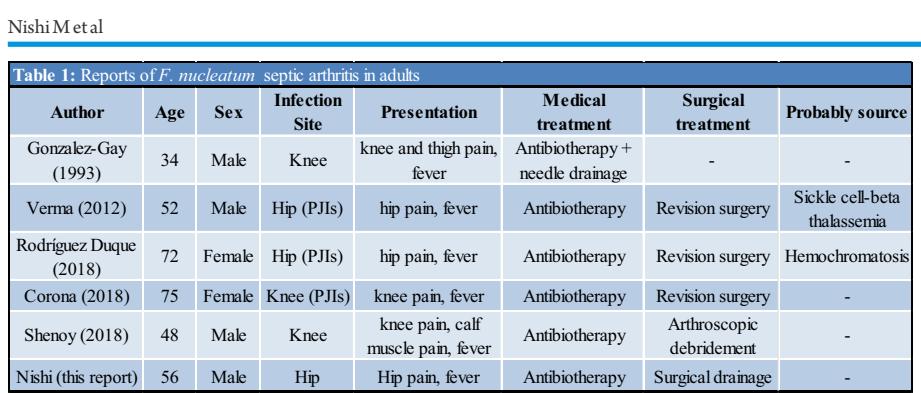

osteoarthritis and a history of Perthes' disease, which may have contributed to the development of hip septic arthritis.

The diagnosis of F. nucleatum is sometimes difficult because of the difficulty in isolating the organism. In our case, F. nucleatum was only isolated from the sample when utilizing anaerobic conditions. We recommend that it is necessary to check for anaerobic cultures in patients with septic arthritis, because many physicians may not routinely submit anaerobic cultures of the synovial fluid.

The treatment of hip septic arthritis in adults typically involves antibiotics and the removal of purulent tissue from the affected joint, either by arthrocentesis or surgical drainage [12]. Regarding antimicrobial therapy, $\beta$-lactam agents, clindamycin, and metronidazole are commonly used for treating Fusobacterium-related infections. However, there is evidence of emerging resistance to penicillins, carbapenems, and clindamycin in some Fusobacterium isolates $[13,14]$. In our case, the isolate was resistant to penicillin.

In previous reports regarding the treatment of septic arthritis due to F. nucleatum, four of the five cases were also treated with surgical debridement and antibiotic therapy (Table 1).

Management of septic arthritis by arthroplasty using the present protocol involves two stage total joint replacement in the case of evolutive arthritis and one-stage total joint replacement in case of quiescent arthritis [15]. Since our case involved evolutive septic arthritis, we planned two-stage revision surgery. There are some reports about two-stage revision surgery for deep infection following septic hip arthritis where the first stage involves debridement and lavage of the joint, followed by resection of the femoral head and replacement of cement spacer antibiotics. We initially attempted to preserve the femoral head because the patient's groin pain was not severe; however, eventually, we performed a femoral head resection and two-stage THA. This explains why surgical drainage was performed 3 times.

There are several reports of THA after septic arthritis. Bauer et al. [16] reported that 26 of the 30 cases ( $87 \%)$ of evolutive septic arthritis were successfully followed up for a mean period of 5 years. Chen et al. [17] found a re-infection rate of $14 \%$ and a complication rate of $36 \%$ in 28 cases, at an average follow-up period of 77 months. In this case, there was no implant loosening or infection relapse after 3 years of follow-up, although further follow-up is warranted.

\section{Conclusion}

We treated a rare case of hip septic arthritis due to F. nucleatum with two-stage revision surgery in addition to a prolonged course of antimicrobial therapy based on susceptibility testing. The exact duration of therapy depends on sufficient debridement, the severity of illness, clinical response, and normalization of inflammatory markers. Clinicians should be aware that F. nucleatum could be the etiologic agent of hip septic arthritis in an immunocompetent patient.

\section{Clinical Message}

F. nucleatum can be the cause of septic arthritis, even in an immunocompetent patient, requiring surgical debridement, and appropriate antibiotic therapy. As with other hip septic arthritis in adult, two-stage revision surgery is effective.

\section{References}

1. Brennan CA, Garrett WS. Fusobacterium nucleatumsymbiont, opportunist and oncobacterium. Nat Rev Microbiol2019;17:156-66.

2. González-Gay MA, Sánchez-Andrade A, Cereijo MJ, Pulpeiro JR, Armesto V. Pyomyositis and septic arthritis from Fusobacterium nucleatum in a nonimmunocompromised adult. J Rheumatol 1993;20:518-20.

3. Laurencet ME, Rosset-Zufferey S, Schrenzel J. Atypical presentation of Lemierre's syndrome: Case report and literature review. BMC Infect Dis 2019; 19:868.
4. Mathews CJ, Weston VC, Jones A, Field M, Coakley G. Bacterial septic arthritis in adults. Lancet 2010;375:84655.

5. Gigante A, Coppa V, Marinelli M, Giampaolini N, Falcioni D, Specchia N. Acute osteomyelitis and septic arthritis in children: A systematic review of systematic reviews. Eur Rev Med Pharmacol Sci 2019;23:145-58.

6. Duque JC, Rubín PG, Humara BG, Sanz AA, Vallina MB, Fernández-Sampedro M. Fusobacterium nucleatum prosthetic hip infection: Case report and review of the literature of unusual anaerobic prosthetic joint infection. 
Anaerobe 2018;54:75-82.

7. Corona PS, Lung M, Rodriguez-Pardo D, Pigrau C, Soldado $\mathrm{F}$, Amat $\mathrm{C}$, et al. Acute periprosthetic joint infection due to Fusobacterium nucleatum in a non-immunocompromised patient. Failure using a debridement, antibiotics+implant retention approach. Anaerobe 2018;49:116-20.

8. Verma K, McNabb PC, Kurtz W, Green J, Trabue CH. Fusobacterium nucleatum prosthetic hip infection in an adult with sickle cell-beta thalassemia. Infection $2012 ; 40: 335-7$.

9. Shenoy PA, Gupta R, Vishwanath S, Naik MA, Shetty S, Chawla K. Parvimonas micra and Fusobacterium nucleatum septic arthritis: A rare anaerobic double trouble. Asian J Pharm Clin Res 2018;11:5-6.

7. Cubero CC, Red PZ, Maestrojuan JJ, Prieto MD. Septic arthritis due to Fusobacterium nucleatum in an immunocompetent patient. Reumatol Clin 2012;8:98-9.

8. Chryssagi AM, Brusselmans CB, Rombouts JJ. Septic arthritis of the hip due to Fusobacterium nucleatum. Clin Rheumatol 2001;20:229-31.

9. Koornstra JJ, Veenendaal D, Bruyn GA, de Graaf H. Septic arthritis due to Fusobacterium nucleatum. Br J Rheumatol 1998;37:1249.

10. Lemierre A. On certain septicaemias due to anaerobic organisms. Lancet 1936;227:701-3.

11. Williams MD, Kerber CA, Tergin HF. Unusual presentation of Lemierre's syndrome due to Fusobacterium nucleatum. J Clin Microbiol 2003;41:3445-8.

12. Sharff KA, Richards EP, Townes JM. Clinical management of septic arthritis. Curr Rheumatol Rep 2013;15:332.

13. Bennett KW, Eley A. Fusobacteria: New taxonomy and related diseases. J Med Microbiol 1993;39:246-54.

14. Brook I. Microbiology and management of joint and bone infections due to anaerobic bacteria. J Orthop Sci 2008;13:160-9.

15. Romanò CL, Romanò D, Meani E, Logoluso N, Drago L. Two-stage revision surgery with preformed spacers and cementless implants for septic hip arthritis: A prospective, non-randomized cohort study. BMC Infect Dis 2011;11:129.

16. Bauer T, Lacoste S, Lhotellier L, Mamoudy P, Lortat-Jacob A, Hardy P. Arthroplasty following a septic arthritis history: A 53 cases series. Orthop Traumatol Surg Res 2010;96:840-3.

17. Chen CE, Wang JW, Juhn RJ. Total hip arthroplasty for primary septic arthritis of the hip in adults. Int Orthop 2008;32:573-80.

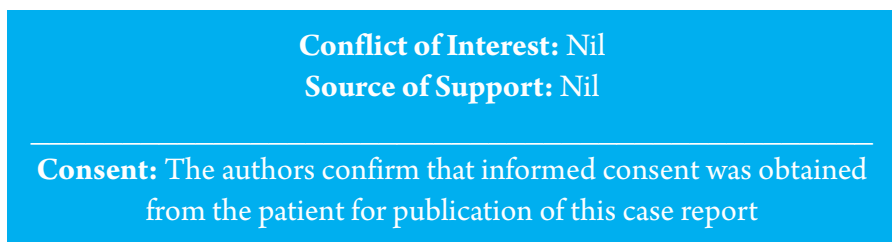

How to Cite this Article

Nishi M, Yoshikawa Y, Kaji Y, Okamoto S, Inagaki K. Isolated Septic Arthritis of the Hip Due to Fusobacterium nucleatum in An Immunocompetent Adult: A Case Report and Review of the Literature. Journal of Orthopaedic Case Reports 2021 April;11(4):37-40 